\title{
Análisis de la educación universitaria agropecuaria en Argentina a través de los periodos de desarrollo
}

\section{Agricultural college education analysis in Argentina through development periods}

\section{Análise da educação agropecuária universitária na Argentina através dos períodos de desenvolvimento}

\author{
Gabriela Civeira* \\ Fecha de recepción: 30 de noviembre 2016 \\ Fecha de evaluación: 5 de septiembre de 2017 \\ Fecha de aceptación: 5 de octubre de 2017 \\ Fecha de publicacion en linea: 1 de diciembre de 2017 \\ DOI: http://dx.doi.org/10.18359/reds.2601
}

Cómo citar este artículo:

Civeira, G. (2017). Análisis de la educación universitaria agropecuaria en Argentina a través de los periodos de desarrollo. Revista Educación y Desarrollo Social, 11(2), 70-89. DOI: org/10/18359/reds.2601.

Ingeniera Agrónoma y doctora en Investigación Agraria y Forestal, Instituto de Suelos Instituto Nacional de Tecnología Agropecuaria (INTA), Buenos Aires, Argentina. Correo electrónico: civeira.gabriela@inta.gob.ar 
Análisis de la educación universitaria agropecuaria en Argentina a través de los periodos de desarrollo

\title{
Resumen
}

Las primeras facultades de agronomía, desde finales del siglo XIX y hasta la crisis de 1930, se fundaron en la región pampeana y litoraleña ambas abastecedora de los productos de exportación que caracterizaban la ubicación de la Argentina en la división internacional del trabajo de la época. Durante la década de 1970, existió el impulso al modelo departamental, el cual fue adoptado parcialmente por algunas universidades que impartían la carrera de agronomía. La formación que se requería en la década del 1990 debía formar a las personas para una sociedad con otros requerimientos, la modalidad más frecuente utilizada para cambiar los planes de estudio se centró en la reestructuración, resultando en una reubicación, aumento o disminución de materias. Los planes de estudio de agronomía evolucionaron desde privilegiar la adquisición de información, a una concepción constructivista tendiente al cambio de rol del alumno, de sujeto pasivo a sujeto activo.

Palabras clave: historia educación agraria, ciencias agrarias, características educación agraria universitaria.

\begin{abstract}
The first agriculture faculties, since the late XIX century and until the 1930's crisis, were founded in the Pampeana and Litoraleña regions, both export producers characterizing Argentina's position in international workforce at the time. During the 70's, there was the impulse to the department model, which was then partially adopted by some universities with agricultural majors. The required training for the 90's should educate people for a society with different requirements; the most used model to change the study plans was focused on the restructure. This turned into the relocation, increase, or decrease of subjects. The study plans for agriculture evolved from awarding the acquisition of knowledge to a constructivist concept towards the students' roles, from the passive to the active student. The current article recommends achieving meaningful and contextualized curriculums for the students, which builds a bridge between the local knowledge and the abroad ones without affecting the identity in each region.
\end{abstract}

Keywords: Agrarian history education, agrarian sciences, college agrarian education characteristics.

\section{Resumo}

As primeiras faculdades de Agronomia, desde o final do século XIX e até a crise de 1930, foram fundadas na região da Pampa e zonas litorais, ambas fornecedoras dos produtos de exportação que caracterizaram a localização da Argentina na divisão internacional do trabalho da época. Durante a década dos 70, houve o impulso para o modelo departamental, que foi adotado parcialmente por algumas universidades que deram a carreira de Agronomia. A formação necessária na década dos 90 devia treinar as pessoas para uma sociedade com outros requisitos; a modalidade mais frequente usada para mudar os planos curriculares foi focada na reestruturação, o que resultou em uma recolocação, aumento ou diminuição de matérias. Os planos curriculares de agronomia evoluíram a partir do fato de privilegiar a aquisição de informações a uma concepção construtivista tendendo a mudar o papel do aluno, de sujeito passivo a sujeito ativo. $\mathrm{O}$ presente artigo recomenda atingir planos curriculares significativos e contextualizados para os estudantes, que construa uma ponte entre o conhecimento dos saberes locais e os universais, sem erodir a identidade de cada região. Essas características até agora foram difíceis de incluir nos planos curriculares.

Palavras-chave: história educação agrícola, ciências agrícolas, características educação agrícola universitária. 


\section{Educación para la construcción del Estado-nación}

Durante la etapa de construcción de las naciones en América Latina se consolidó el Estado como una organización donde si bien el modelo favoreció el crecimiento paulatino de los sectores medios y una mayor heterogeneidad social y cultural (Tedesco, 1986), solo tuvieron la posibilidad de participar en los Gobiernos cada uno de los países de América Latina y en la economía un sector dirigente que presentaba un gran poder frente al grupo dirigente. Este sector estaba integrado por el conjunto de los productores agropecuarios, exportadores y los integrantes del sistema financiero que proveían el mercado internacional. Argentina, al formar parte de una etapa denomina modernización temprana (Filmus, 1999), presentó una acotada etapa colonial en la cual existió una elevada y heterogénea inmigración de pobladores de origen europeo que formaron parte del sector rural y urbano.

Debido a esto el modelo productivo estuvo obligado a incluir un gran volumen de mano de obra, proveniente de la inmigración (Filmus, 1999). Si bien el modelo permitió un poco de autonomía a los ciudadanos, estos grupos no pudieron acceder a la participación política; asimismo, en el plano político y económico tampoco permitió el acceso masivo a la propiedad y a la movilidad 72 social ascendente. En este marco, el sistema educativo fue regulado y conformado por el Estado y mediante la educación logró un mecanismo óptimo para homogeneizar y renovar a la sociedad del momento histórico (Tedesco, 1986). El Estado nacional asumió el rol de controlar la educación mediante la gestión propia o a través del control de las instituciones de tipo privado; además, buscaba homogeneizar a una población desigual y heterogénea. En sus orígenes, el sistema educativo estuvo relacionado con el ámbito político y no con el económico. En este momento histórico, la educación estuvo enfocada a lograr la integración social, consolidar la identidad nacional y construir el Estado (Tedesco, 1986).

Esta etapa se caracterizaba por la explotación agropecuaria extensiva y una industria rudimentaria que no requería mano de obra con una calificación técnica específica. Asimismo, la baja demanda de mano de obra calificada pudo ser satisfecha por obreros que provenían de Europa y que ya conocían el oficio. Por lo tanto, en general los Gobiernos no fomentaron la capacitación en mano de obra calificada, con lo cual no fueron incluidos en el currículo oficial (Puiggrós, 1990). La estructura escolar estuvo pensada para generar un "sistema de estratificación social" (Filmus, 1999) que fuera útil para el Gobierno. La idea básica era impartir contenidos culturales homogéneos que permitieran la formación en la identidad nacional en los primeros años de escolaridad. Por otro lado, la enseñanza superior o media no permitía el acceso a toda la población, no presentaba un objetivo tan claro y solamente capacitaba para la administración pública, a su vez que 
seleccionaba y habilitaba a una élite para incorporarse posteriormente a la dirigencia política y al Estado (Filmus, 1999). Tanto la estructura educativa como el currículo estaban fuertemente gobernados por el Estado: concentrados y generados desde la provincia de Buenos Aires. Esto contribuyó a que ese modelo de país agroexportador (de la década de los 80) se legitimara y expandiera rápidamente por toda la Argentina.

Esta etapa de conformación del Estado nacional,(en Argentina en particular) estuvo sumergida en una contradicción entre los ideales del liberalismo en sus manifestaciones, cuyos principios educativos estuvieron planteados en parte en la Constitución Nacional y en la Ley 1420 y un modelo político, económico y social que, a pesar de mostrarse modernizador en varios aspectos, no logró incorporar a grandes sectores de la población argentina (Filmus, 1999). La apertura en las oportunidades educativas si bien permitió que una importante cantidad de los sectores medios accediera a la escolaridad media y superior, también ayudó a que estos grupos comenzaran a reclamar un proceso de democratización en la estructura gubernamental. Asimismo, a pesar de los grandes esfuerzos realizados para alcanzar el incremento en la inclusión educativa, por ejemplo a través de la alfabetización para inmigrantes y la apertura de escuelas rurales, finalmente no se logró que los sectores más postergados de la población pudieran acceder y permanecieran en el sistema (Tedesco, 1986). Además, el sistema presentaba grandes desigualdades educativas entre las diferentes regiones del país, lo que hizo, entre otras causas, que el Estado tuviera grandes dificultades para asegurar las funciones del sistema educativo. El Estado benefactor que se instaló en el periodo posterior desempeñaría varias de estas funciones, aunque algunas aún hoy no han sido llevadas a cabo y se mantienen como deudas pendientes hacia una gran parte de la población (Filmus, 1999; Tedesco, 1986).

En este tiempo, la economía nacional estaba basada en el sistema agropecuario de la región pampeana, el cual realizaba un uso exhaustivo del abundante recurso suelo distribuido en áreas con características agroclimáticas que privilegiaban su desarrollo. Este modelo fue altamente dependiente de los recursos naturales y con el avance científico tecnológico fue perdiendo competitividad internacional en las etapas posteriores. El sistema económico-agropecuario estaba pensado para producir productos agrícolas y ganaderos que pudieran satisfacer la demanda internacional (Instituto Interamericano de Cooperación para la Agricultura [IICA], 1998). Por otro lado, estaban las economías regionales circunscritas desde el nordeste hasta la Patagonia, que se constituyeron durante la colonización española y que presentaban un perfil agropecuario no dominante basado en producciones intensivas y agroindustriales con una elevada contratación de mano de obra y principalmente relacionada con el consumo interno y alejado de los puertos del litoral. El modelo de país con énfasis en el agro pampeano creó fuertes inestabilidades regionales 
que originaron la pérdida de importancia e incluso marginalidad de este tipo de economías. Este proceso recién empezó a cambiarse durante la década de los 40 cuando se adoptó el modelo de sustitución de las importaciones que generó un aumento en la demanda de alimentos del mercado interno.

El origen de los estudios en agronomía en la Argentina, hacia fines del siglo pasado, se supeditó a las demandas que provenían del contexto donde se desenvolvía el sector agropecuario en esa época. Durante esta etapa se crea el Instituto de Santa Catalina y comienzan los estudios agronómicos en el país. En 1870, se forman departamentos de enseñanza de la Agronomía en los colegios nacionales de Salta, Tucumán y Mendoza. En 1883, comienza la enseñanza superior mediante la creación del Instituto Agronómico y Veterinario de Santa Catalina en Buenos Aires. De esta manera, se crea la primera Facultad de Agronomía en la Universidad Nacional de La Plata y al comenzar este siglo (en 1904) se funda la de la Universidad de Buenos Aires, que en conjunto con la de la Universidad Nacional del Nordeste fueron las únicas ofertas académicas, hasta que en la década de los 40 se crean las Facultades de Ciencias Agrarias de Cuyo y de Agronomía y Zootecnia de Tucumán y el Departamento de Agronomía de la Universidad Nacional del Sur, en Bahía Blanca (IICA, 1998).

Este hecho no es casual debido a que el proyecto nacional de la Generación del 80 se constituyó sobre el desarro- llo agropecuario ligado a la principal potencia de la época que era Inglaterra. De ese mismo país provenían los ferrocarriles, el capital, los puertos y la principal demanda de los productos agropecuarios. Consecuentemente, se intentaba no solo poblar, sino también integrar e insertar al país en el mundo. Se promovió la inmigración, se expandieron las vías férreas y se logró ocupar el territorio productivamente (Barsky, 1988). El país ya era un gran productor de carne, granos y lana; por lo tanto, los estudios necesarios se planteaban para aportar conocimientos y profesionales para ese sector agropecuario. La carrera de Agronomía fue creada para servir a esa demanda en dicha época y en ese proyecto de país. Este modelo de Estado unido al sistema agropecuario se prolongó por más tiempo que el de la vigencia de ese modelo y determinó en gran medida el perfil de los profesionales de las Ciencias Agropecuarias por varias décadas.

\section{Educación para el crecimiento económico: el Estado benefactor}

En esta etapa, debido a los cambios producidos en el ámbito internacional, fue necesario cambiar los patrones del crecimiento económico. A partir de la crisis del 30 y de la Segunda Guerra mundial, se sucedió la caída de las exportaciones de materias primas y de las importaciones manufactureras, lo que obligó a desarrollar una industria que pudiera sustituirlas. Sin embargo, 
no existían en la sociedad sectores con la capacidad económica y la decisión política para llevar a cabo este proceso de industrialización; por esta razón, el estado tuvo que tomar esta iniciativa y conducir esa nueva etapa. Esta modificación de los requerimientos educativos del aparato productivo logró transformar también las funciones principales del sistema educativo.

En la Argentina el Estado de bienestar recién se iba a desarrollar plenamente cuando asumiera el Gobierno peronista (1945). En esta etapa existieron golpes de Estado mediados por los sectores tradicionales que representaban a un grupo con poder concentrado, los cuales intentaban volver al modelo hegemónico que finalmente no pudo reestablecerse en esta nueva etapa industrial donde se había logrado incluir a la población en las industrias que estaban creciendo (Filmus, 1999). Esta nueva forma de Estado tuvo características comunes en toda Latinoamérica, en lo económico profundizó el carácter intervencionista y anuló el libre mercado propuesto por el capitalismo. Además, intentó regular el mercado mediante una planificación que pretendió mejorar la economía y desarrollar el sector público en las áreas productivas y de servicios públicos. Fomentó a los sectores industriales el desarrollo del mercado interno; permitió la incorporación de otros sectores al Gobierno y redistribuyó recursos en estos sectores conformados por los asalariados, y de esta manera generó una mayor homogeneidad en la socie- dad colaborando en el acceso a mejor calidad de vida. Este tipo de Gobierno fue conocido como Estado benefactor.

En esta etapa también existió la intervención del Estado en el sistema educativo, pero uno de sus fines era lograr incorporar a nuevos sectores a la participación social y laboral. Por lo tanto, el sistema educativo necesitaba incorporar a un mayor número de estudiantes y aumentar la matricula en las escuelas. En este marco la educación significaba un derecho de los ciudadanos y una estrategia de capacitación de mano de obra para satisfacer las demandas de la industria. La antigua "formación del ciudadano" que caracterizó al periodo anterior fue reemplazada por la idea de "formación para el trabajo" que luego, a partir de las teorías del capital humano, se denominaría "formación de recursos humanos" (Filmus, 1999; Tedesco, 1986). Los nuevos tipos ocupacionales demandaban una alfabetización básica que el sistema educativo estaba obligado a ofrecer (Tenti Fanfani, 2001). Se comenzó a demandar ciertos niveles técnico-profesionales, conocimientos de oficios y especialidades que no alcanzaban con ser aprendidos en el lugar de trabajo y que los nuevos trabajadores, a diferencia de los inmigrantes, no poseían. Se demandaba una disciplina laboral que solamente el sistema educativo podía brindar intensivamente a millones de trabajadores rurales que procedían del interior del país y que iban a formar la principal fuerza laboral de los nuevos talleres y fábricas (Carciofi, 1987). 
A finales de la década de los 40 empezó a generarse una visión diferente del papel de la educación, de la mano de las teorías de capital humano; dejó de ser vista como un gasto social para transformarse en una inversión que tenía como principal objetivo alcanzar un ingreso tanto individual como social. Los factores que eran considerados tradicionales para la economía clásica no eran suficientes para el crecimiento de los países; por lo tanto, la capacidad de generar "capital humano" o mano de obra con una elevada calificación comenzó a ser una de las mejores ventajas comparativas para el crecimiento de los Estados a nivel internacional y regional. Esta concepción se profundizó con el surgimiento del modelo de Estado desarrollista, luego del derrocamiento del presidente Perón (Filmus, 1999, Tedesco, 1986, Tenti Fanfani, 2001).

Este modelo fue definido como un tipo del Estado benefactor, pero que centró su acción en la promoción y conducción del desarrollo económico. Estas concepciones económicas de la educación como inversión permanecieron hasta mediados de la década de los 70, cuando ya se encontraba avanzada la crisis del Estado desarrollista. Existió una nueva alianza conformada por sectores empresariales vinculados al capital extranjero como bancos, productores de bienes exportables primarios y grupos tecnocráticos,

76 quienes pensaban que la participación de los sectores populares, como en las décadas anteriores, perjudicaba al modelo de acumulación formulado.
En el ámbito educativo se manifestaban discursos modernizantes y tecnocráticos en torno a la universalización y el papel de la educación en el crecimiento y las políticas a través de las cuales el Estado comenzó a desatender la distribución social de conocimientos a través de la escuela. Los efectos de este modelo se comenzaron a manifestar mediante el deterioro de la calidad educativa ofrecida por el sistema. Los recursos destinados a las políticas educativas no se incrementaron, mientras que la demanda por educación siguió creciendo y se amplió la matrícula escolar en todos los niveles. Por otra parte, existieron elevados mecanismos de planificación educativa que fueron utilizados con el objeto de mejorar la contribución de la educación a las estrategias de crecimiento del país. Estas discrepaban con una realidad donde los actores económicos y sociales mostraban sus propias lógicas de comportamiento. Finalmente, en 1966 ocurrió una intervención de las universidades, lo que contribuyó al recorte en los contenidos curriculares, a la expulsión de maestros, y esto llevó a que muchos investigadores tuvieran que abandonar el país, lo cual generó una profunda contradicción en este modelo (Rama, 1987; Filmus, 1999, Tedesco, 1986).

Las primeras facultades de Agronomía, desde finales del siglo XIX y hasta la crisis de 1930, se fundaron en la región pampeana y litoraleña, ambas abastecedoras de los productos de exportación que caracterizaban la ubicación de la 
Argentina en la división internacional del trabajo de la época. El siglo XX se inauguró con la competencia entre naciones capitalistas europeas que produjo la Primera Guerra Mundial y con la respuesta a las consecuencias sociales del capitalismo que, bajo las banderas del socialismo, condujo a la Revolución rusa. En estos sucesos políticos estaba inmersa la Argentina agropecuaria. Recién en la década de los 40 se crearon las facultades de Cuyo y Tucumán que se corresponden cuando se desarrolla fuertemente el modelo económico de sustitución de importaciones, con especial hincapié en el consumo interno, del que esas economías regionales eran importantes proveedoras. Nuevamente esta recuperación demuestra la importancia que ejerce la demanda del sistema económico en la estructura productiva (IICA, 1998, Barsky, 1988). Por lo expresado anteriormente, parecería que la razón de más peso que motivaba la generación de las universidades con carreras agronómicas era la económica y no la necesidad de creación de nuevos espacios académicos.

La disolución del modelo autoritario ocurrido en 1973 y la recuperación de la democracia coincidieron con el comienzo del ocaso del estado benefactor. El periodo de expansión del modelo de Estado benefactor concluiría sin que las promesas realizadas en relación con las funciones del sistema educativo fueran plenamente alcanzadas. Si bien la escolaridad se expandió fuertemente, aun en 1970 continuaban las altas tasas de deserción y las desigualdades entre zonas geográficas. Los factores que desencadenaron la crisis de este modelo de Estado burocrático autoritario tecnicista y racional fueron las movilizaciones de sectores sociales excluidos que reclamaban la restauración de una democracia que los incluyera (Filmus, 1999).

\section{Educación como función política: crisis del Estado benefactor}

En el marco de grandes crisis fiscales de la década de los 70 y el aumento de los precios del petróleo, el Estado benefactor no pudo sostener políticas redistributivas y no era posible sortear la crisis mediante la intervención más profunda por parte del Estado. A nivel mundial, comenzó un proceso de gran pesimismo respecto del aporte de la educación a la economía y se hizo énfasis en las teorías de la educación en torno a la reproducción de las desigualdades socioeconómicas. En la Argentina, los diferentes Gobiernos que administraron el Estado en este periodo priorizaron la función política de la educación y disminuyeron así su rol económico. Esta disminución del rol económico de la educación comenzó poco antes de la crisis de crecimiento que existió a partir de 1975 (Filmus, 1999, Tedesco, 1986).

\section{Gobernar}

Durante esta etapa se generaron las condiciones para que la crisis del Estado 
benefactor fuera irreversible, y la estrategia que se adoptó fue la limitación de los recursos destinados a las políticas sociales. A pesar de haber sido uno de los objetivos planteados, no se mejoró la calidad de los servicios brindados por el Estado y el aparato estatal presentó ineficiencia y alta burocratización. Tampoco se logró el proceso de privatizaciones ni el abandono completo de la participación del Estado en el marco económico. Además, la modernización de la administración pública no fue lograda y la corrupción y la especulación financiera, tanto en el ámbito público como privado, fue una de las características predominantes en todo esta época. El periodo desde 1976 hasta 1983 dejó una deuda externa e interna, crisis fiscal y proceso inflacionario que debió enfrentar el Gobierno democrático que asumió en 1983. La concentración del poder económico en un sector, la disminución del poder de los sectores obreros y la nueva crisis que experimentaron los sectores bajos y medios lograron fomentar los conflictos económico políticos que existieron durante dicho Gobierno (Filmus, 1999).

En un primer periodo, el papel de la educación en relación con la economía, cuando aún no había declinado el crecimiento, se desvalorizó. La función política de la educación estuvo reforzada en su papel ideológico delimitando como principal objetivo la liberación nacional, lo cual implicaba la nacionalización de la educación para la construcción e integración a la sociedad y los valores de la comunidad. Esta función ideológica también alcanzó a la universidad estatal y privada, donde se planteaba en su enseñanza la doctrina nacional y se buscaba "impedir la infiltración del liberalismo, del positivismo, del historicismo, del utilitarismo y de otra penetración ideológica en las casas de estudio" (como lo señalaba el rector de la Universidad de Buenos Aires [UBA], Puiggrós, 1973). En una segunda etapa, la educación hizo posible conseguir el orden social imperativo para poder realizar las transformaciones planteadas. La combinación de las políticas permitió extraerle a la escuela los contenidos socialmente significativos y los métodos de construcción del conocimiento necesarios para la participación social. Comenzó otra forma de socializar a niños y jóvenes, con mayores niveles de autoritarismo, jerarquización y discriminación; el orden y la disciplina fueron mucho más importantes que el proceso de enseñanza-aprendizaje. Cabe destacar que en este periodo se produjo la transferencia de las escuelas primarias a las jurisdicciones con un claro objetivo economicista de la descentralización. Las consecuencias más relevantes fueron la diminución en la calidad educativa y en el aumento de su heterogeneidad cualitativa, las cuales habían sido eliminadas del sistema educativo argentino durante las primeras etapas (Tedesco, 1986).

Durante esta nueva fase, el perfil profesional del agrónomo no fue modificado porque tampoco estuvo cuestionado su lugar en el desarrollo agropecuario; se 
incorporaron nuevos conocimientos a una matriz que permanecía inalterada. Las reformas posteriores cambiaron los planes de estudio, pero no debatieron la estructura curricular vigente, solamente incorporaron los nuevos avances tecnológicos, como la amplificación de la revolución industrial al agro mediante la mecanización y el uso de agroquímicos y el desarrollo de la revolución verde con los avances en la genética. La razón de no cambiar el perfil es que no había cambios en el sistema agropecuario, las metas del sector eran cuantitativas y la respuesta profesional era también cuantitativa, o sea acumulativa en conocimientos. La formación estaba basada solamente en disciplinas que se sucedían y repetían el modelo "fordista" similar al que prevalecía en modelo industrial, debía dar respuesta a problemas que habrían de repetirse en el futuro. Los desarrollos intelectuales se limitaban a pensar en un lote, un cultivo o un establecimiento. Las partes eran comprendidas separadamente y se intentaba unirlas en un todo mediante superposición. Esta era una realidad marcadamente estancada y generaba la formación de un ingeniero agrónomo por sectores "de abajo para arriba y de adentro para afuera” (IICA, 1998).

Un cambio importante a nivel universitario, durante la década de los 70, fue el impulso al modelo departamental, adoptado parcialmente por algunas universidades que impartían la carrera de Agronomía (por ejemplo, la Universidad Nacional de Lujan y la Universidad Nacional del Sur). Este modelo ha demostrado la existencia de tensión que se debió particularmente al sistema de designación por materias que caracterizaba al modelo de cátedras y que finalmente bloqueaba los intentos de introducir reformas en los planes de estudio de Agronomía y de otras carreras.

La asunción del Gobierno democrático (1983) comenzó otro periodo, que tuvo que administrar la etapa más crítica y tal vez la última del Estado de bienestar que había surgido a partir de mediados de la década de los 40. Durante este tiempo la crisis que había comenzado en la década anterior no pudo ser revertida, en parte debido a la herencia y a fallas en la administración pública. Existió entonces disminución de la producción, menor inversión, caída en la contratación de mano de obra, elevada inflación y concentración económica en pocos sectores. La educación tuvo una función política debido a que debió desarmar el orden autoritario a través de una formación más democrática. El sistema educativo generó una vuelta al papel originario del siglo XIX para lograr aumentar la participación ciudadana: permitió abrir centros de estudiantes, reincorporó docentes, mejoró el ingreso a las universidades, cambió planes de estudio y logró normalizar las universidades estatales, entre otras modificaciones que fueron desarrolladas (Braslavsky y Tiramonti, 1990).

Este Gobierno democrático generó espacios para la discusión, como el Congreso Pedagógico Nacional; sin embargo, no consiguió implementar los acuerdos allí 
alcanzados; la intervención estatal en la realidad escolar fue solo parcial, no estuvo acompañada de políticas educativas dirigidas a retomar su responsabilidad en torno a brindar reales posibilidades de acceso a una educación de calidad para todos los argentinos. Se modificaron las formas, pero manteniendo los contenidos, los actores del proceso educativo expresaron su disconformidad con la falta de atractivo y significación social de los conocimientos escolares. Existió ausencia de transformaciones profundas en los procesos de enseñanza aprendizaje en la escuela, lo que llevó a imponer nuevamente el orden educativo autoritario para disminuir las reacciones adversas de los estudiantes secundarios (Filmus, 1999). Esto último, sumado a condiciones socioeconómicas desfavorables, contribuyó a generar una creciente desaprobación de la sociedad en relación con este modelo democrático y un nuevo tipo de Estado empezaba a ocupar el centro de la escena. En este nuevo escenario hubo que redefinir cuál sería la función que debía desempeñar la educación.

\section{Educación como bien de intercambio: el Estado postsocial}

\section{Fines de los 80 y principios de los 90}

80

Hacia finales de 1980 el Estado benefactor se encontraba en crisis debido a la tendencia hacia la globalización que disminuyó la capacidad de decisión del Estado nacional. El mundo avanzaba hacia "un solo mercado de bienes, servicios, tecnología y capital" (García Delgado, 1994) y los flujos financieros eran cada vez más dinámicos. Las limitaciones a la intervención del Estado no solo estaban impulsadas desde factores internos, sino también por factores externos, como los organismos financieros internacionales (García Delgado, 1994). El deterioro del Estado de bienestar en los países latinoamericanos generó una transformación en este (denominado de diferentes maneras: postsocial, neoliberal, democrático-liberal, etc.). Dicho Estado abandona su papel interventor en la economía al comenzar a privatizar empresas, reducir el empleo estatal y el gasto público, aumentar la capacidad de recaudación impositiva, descentralizar varias de sus funciones y transferir al mercado la conducción del modelo de desarrollo y de distribución de bienes, con lo que permitía la acumulación orientada a la competitividad externa más que al mercado interno. Es un Estado que se encoge, y que transfiere así responsabilidades hacia el mercado y la sociedad. La concentración del poder económico, el aumento de la desigualdad social, el incremento de la tasa de desocupación y los cambios en los comportamientos sociales, respecto al cumplimiento de las normas, inclusive las legales, fueron algunas de las principales consecuencias del proceso. Los cambios producidos en la estructura y el papel del Estado en la nueva relación que plantea este con la sociedad influyen en las funciones de la educación. Una de 
las transformaciones más relevantes fue la nueva relación que se plantea entre la educación, la estructura productiva y el mercado de trabajo (Filmus, 1999).

Las transformaciones de la década de los 90 han cambiado varios aspectos del contexto político, económico, social y educativo. Estos cambios también han ocurrido en la matriz tecnológica productiva del sistema agropecuario argentino. Las nuevas tendencias en el sector agropecuario y los cambios en el mercado mundial aumentaron la presión de uso sobre los recursos naturales debido al crecimiento de demanda de productos agroalimentarios hacia países en vías de desarrollo. Este aumento en la demanda estuvo también asociado a la aparición de nuevos cultivos y tecnologías provenientes de la agroindustria internacional que desembarcó en el país a mediados de la década de los 90. Este modelo agroexportador determinó la estructura social agraria con diferentes actores económicos y aumentó las diferencias entre productores, al aumentar el nivel de dependencia de los que presentaban menor capacidad de negociación. A la vez, existió una disminución en el mercado interno y externo para las materias primas y los alimentos, lo que generó cambios en las estrategias productivas, financieras, comerciales y en la adopción de tecnologías por parte de los productores agropecuarios (Balsa, 1993). Por lo tanto, la situación de la producción familiar se vio severamente afectada generando el abandono de las tierras en varias provincias y la disminu- ción de la participación en el mercado (Craviotti, 2000).

Lo anterior, sumado a los cambios en la matriz científico-tecnológica, la disminución en el interés en la producción agropecuaria y la menor importancia de las producciones primarias debido a los cambios estructurales de la economía generaron que la carrera de Agronomía en el país se encontrara en un estado de cuestionamiento, especialmente en cuanto a que estos sistemas educativos eran obsoletos para los futuros profesionales agropecuarios (Barsky y Davila, 2002). En esta etapa se planteaba que la educación necesitaba menos conceptos y más procedimientos, actitudes y valores. Esto involucró cambios en los contenidos y en la organización educativa en el nivel medio (en el que se incluía a la educación agrotécnica). La educación obligatoria se extendió a 10 años, seguida del nivel polimodal por 3 años y del programa los Trayectos Técnico-Profesionales (TTP) (ambos no obligatorios). La Ley Federal de Educación (Ley 24195) planteó una profunda transformación del sistema educativo que pretendía hacer frente a los cambios ocurridos en la década de los 80. La sociedad estaba caracterizada por un modelo de trabajador y ciudadano que requería educar para la disciplina en un caso y la libertad en el otro. Estos fueron derivando hacia formas de organización social diferentes. La formación que se necesitaba en la década de los 90 debía formar a las personas para una sociedad con otros requerimientos. Estas grandes modificaciones 
en el nivel medio, del que provienen los ingresantes a la universidad, tuvieron influencia a mediano plazo en su formación e impactaron fuertemente sobre las disciplinas de los primeros años de las carreras universitarias. El nivel polimodal se organizó en varias modalidades y los TTP eran planteados como una capacitación para el trabajo, y para que generaran una orientación a los estudiantes preuniversitarios, y que la universidad debería tomar en cuenta para sus diseños curriculares (IICA, 1998). Sin embargo, el mundo del trabajo, con sus características de segmentación, flexibilidad y movilidad, siguió un ritmo diferente que el educativo. El sistema educativo fue planeado para generar una capacitación básica que habilitara para la adaptación y capacitación a nivel laboral. Las grandes transformaciones sociales, culturales, económicas y productivas, especialmente el cambio en la lógica del sistema económico produjo un cambio en la lógica del sistema educativo. Se comenzó a hablar de países competitivos y la regulación de la economía era regida por la lógica del mercado (IICA, 1998, Tedesco, 1986).

La reforma educativa abordó no solo los contenidos, sino también procedimientos, actitudes, y estructuras institucionales. En relación con la articulación entre facultades, el nivel polimodal, los contenidos básicos, los contenidos orientados y el trayecto técnico profe82 sional agropecuario donde se pretendía formar al estudiante, solo se cubrían parcialmente los conocimientos necesa- rios para el ingreso a la universidad. En este sentido, se constituyó el programa TTP de la Dirección Nacional de Educación Técnico-Profesional del Instituto Nacional de Educación Tecnológica (INET), que incluyó un foro agropecuario conformado por algunas entidades educativas, de la producción, gremiales, profesionales y estatales vinculadas con el sector agropecuario, para efectuar los cambios que permitieran incorporar a la enseñanza agrotécnica los lineamientos de la nueva estructura de la educación nacional. La tarea en este marco de participación fue segmentada y complementada con consultas a especialistas que respondían a las necesidades de un sector (IICA, 1998). Asimismo, se realizó una revisión y reorganización del nivel de formación técnico agropecuario, debido a que existía una enorme dispersión, que presentaba muchas escuelas agrotécnicas de diferentes jurisdicciones (aproximadamente 400) y que otorgaban más de 100 títulos diferentes. Se caracterizó el perfil profesional, las capacidades y las competencias que debían cumplir para obtener el título. Se identificaron los puntos de conflicto en la articulación entre facultades y TTP Agropecuario; en este último se remarcaba que era necesario mejorar el aspecto técnico operativo: el egresado debía presentar capacidad de ejecución ("que sepa hacer") (IICA, 1998). En general, se puede afirmar que la implementación del ciclo polimodal no logró los objetivos propuestos debido a que no tuvo relación con las carreras técnicas universitarias, no existió correlación con los ciclos niveladores de 
ingreso universitario y la capacitación de docentes de enseñanza media en las universidades, y tampoco se implementó completamente la incorporación de la problemática científica en el nivel medio.

Como se planteó anteriormente, durante esta etapa el sistema productivo agropecuario experimentó grandes cambios lo que generó que las interrelaciones de los diferentes componentes constitutivos del sistema se tornaran más complejas (Balsa, 1993). Debido a esto, derivaron los problemas de la transformación curricular, donde se comenzó a plantear la imposibilidad de enseñar todo y la necesidad de conservar una concepción integradora al mismo tiempo. En este contexto, la definición del currículo es imprescindible; por lo tanto, algunas condicionantes estructurales del medio agropecuario, como la diversidad de sistemas productivos, la extensión territorial, las características organizativas de las empresas agropecuarias y la movilidad laboral de los profesionales establecieron exigencias que fueron difíciles de solucionar a través de un solo modelo curricular (Balsa, 1993). A lo largo de varias décadas y particularmente en esta etapa, el perfil profesional fue planteado atendiendo a las especificidades que demandaban el mercado y la ciencia, que en general respondía a adaptaciones de tecnologías de países desarrollados. En este sentido, las reformas curriculares en las facultades de Agronomía se desarrollaron bajo la presión de esta complejidad y la diversidad que contiene, e impactaron así en los contenidos mínimos que definieron la identidad de la profesión y establecieron el estándar de calidad imprescindible en los conocimientos del profesional del momento histórico (Lagomarsino y Amado de Fernández, 2012).

Uno de los primeros debates que se planteó fue que ningún profesional podía tener un conocimiento y manejo en profundidad de las temáticas abarcadas en el proceso productivo agropecuario (por ejemplo, desde el uso y conservación de los recursos naturales, pasando por la integración vertical de procesos agroindustriales y las cadenas de distribución de productos). Por lo tanto, académicamente ya no se podía pretender un modelo de ingeniero agrónomo "generalista", con una formación abarcativa de la totalidad de la problemática agropecuaria, como prototipo del perfil profesional (IICA, 1998). Ese modelo tradicional que estaba en vigencia presentó resistencia a cualquier nuevo modelo y potencial "ajuste" de los conocimientos e incumbencias por reducción de temáticas o división del currículo, todo esto era visto como una pérdida del sentido mismo de la agronomía. Dentro de este marco conceptual, se plantearon las cuestiones de base que influyeron en la construcción del perfil profesional "generalista" y que presionaron, tanto desde adentro como desde afuera, para lograr un aumento sobre el currículo de la carrera. Esto condujo a una estructura acumulativa que derivó en el alargamiento permanente de los estudios de grado y que durante este 
periodo se había tornado insostenible. Por otra parte, las obligaciones legales establecían cargas horarias mínimas muy altas (superiores a 3500 horas), lo que obligaba a estructurar carreras de muy larga duración. Ese carácter totalizador no permitía implementar el debate sobre la adecuación de las carreras a tiempos caracterizados por la gran producción de conocimientos y la velocidad de su obsolescencia (IICA, 1998; Lagomarsino y Amado de Fernández, 2012).

El perfil profesional del agrónomo propuesto en esta etapa tuvo el objetivo de generar una visión sistémica u holística que era planteada como la comprensión del complejo proceso de la producción agropecuaria y a su vez debía responder a la demanda de la sociedad del momento. El debate sobre el perfil del agrónomo incluía también la discusión sobre dividir la carrera en varias carreras de grado o transformarla en un tronco común básico que se ramificara en varias especialidades. En este sentido, cualquiera de estas alternativas significaba la desaparición del ingeniero agrónomo "generalista", totalizador, supuestamente preparado para atender cualquier requerimiento de la amplia realidad agraria que formaba para cualquier situación que se presentara y daba la imagen de una mayor diversidad y, supuestamente, flexibilidad (IICA, 1998). Uno de los primeros temas planteados fue acortar las carreras para lograr egresados más 84 jóvenes para que pudieran regresar pronto a actualizar su formación, a medida que los conocimientos cambiaran. En ese momento se incluyó en el debate la propuesta acerca de que lo más importante era lo constitutivo de la agronomía, cuya permanencia debía garantizarse al momento de reducir las temáticas y los contenidos. La disminución en los temas abordados fue vista como el peligro de un profesional de menor calidad, con inexactitudes académicas. Por otro lado, los planteos universales (que existen en todas las profesiones) del modelo de la época estaban basados en la regulación por el mercado y promovían la intensificación de la formación básica e inducían a pensar en un egresado con carencias técnicas para el desempeño laboral en el ámbito productivo. Este problema se expresó como una puja entre la calidad y la cantidad (IICA, 1998; Barsky, 1998).

Varios problemas fueron señalados como los causantes del retraso y la reducción de la posibilidad de realizar cambios profundos en la oferta curricular: la incidencia de las materias básicas generales sobre la estructura de toda la carrera, el elevado peso de los tres primeros años y la poca posibilidad de modificarlos, el antagonismo entre la necesidad de incentivar esa formación (acentuando su peso) y la de cubrir carencias en áreas relacionadas con el desarrollo profesional; la falta de vinculación entre contenidos y metodologías de enseñanza; la multiplicación de facultades de Agronomía, especialmente en el ámbito privado, con tendencia a la resolución coyuntural de las demandas de nuevos perfiles profesionales, redistribuyó el alumnado y complicó aún más el problema; la 
desarticulación entre los sistemas de enseñanza agrotécnica y agronómica; las resistencias internas en los claustros para realizar cambios; las universidades con estructuras departamentales rígidas en las que los cambios en una carrera tenían impacto en los departamentos académicos que dictaban asignaturas de varias carreras; el desconocimiento de experiencias internacionales en la búsqueda de soluciones al currículo agronómico; la falta de flexibilidad en las opciones curriculares para poder atender al conjunto de las demandas laborales profesionales.

Asimismo, todos estos problemas condujeron a duraciones reales de la carrera de 7-8 años, hecho que generó un mayor número de estudiantes que debían trabajar para mantenerse en el sistema (IICA, 1998, Lagomarsino y Amado de Fernández, 2012).

Los cambios curriculares fueron llevados a cabo teniendo en cuenta el contexto institucional, los ámbitos regional y nacional donde se desenvolvían las instituciones educativas, debido a que en ambos se producían transformaciones de importancia que las afectaron y que influyeron al momento de definir los contenidos de los planes de estudio. En varios países de Latinoamérica se constituyeron comités de decanos (Vicedecanos, Secretarios Académicos) u otras estructuras similares (foro de análisis de la educación superior agropecuaria formado por las autoridades de las universidades, que luego constituyeron la Asociación Universitaria de Educación
Superior Agropecuaria [Audeas], en Argentina), con el propósito llevar a cabo el debate de la problemática de los cambios curriculares.

Uno de los problemas de estos comités fue que al no incluir a todos los actores de la universidad en el debate, los resultados del proceso de reforma fueron sesgados hacia lo que un grupo de poder indicaba. En las nuevas transformaciones curriculares planteadas a nivel continental logró prevalecer y se tuvo en cuenta la lógica de mercado; las temáticas recurrentes que finalmente se incluyeron fueron la creciente competencia de otras profesiones; la necesidad de reducir y de recortar por algún lado; la necesidad de empezar desde las incumbencias laborales y de las habilidades profesionales requeridas; la necesidad de asimilación del cambio tecnológico acelerado que se genera fuera de las universidades y la necesidad de autofinanciamiento. Por lo tanto, la investigación deriva hacia formas de mayor dependencia empresarial, con incremento del nivel práctico o de aplicación y la necesidad de atender la demanda del mercado laboral y no la demanda estudiantil y de los docentes (IICA, 1998).

El siglo XX culmina con el fin de la Revolución industrial, desaparecen los principios de la producción masiva uniforme y, en serie, los límites precisos de los Estados nacionales, el uso indiscriminado de la energía y los recursos naturales, las ventajas comparativas de los recursos y la indiferencia ambiental. El siglo estuvo dominado por el desarrollo, 
con todas sus consecuencias sociales, económicas, políticas, tecnológicas y ambientales. El contexto estuvo dado por los cambios estructurales que la economía, la producción y el comercio tuvieron a nivel nacional y mundial por los avances de una gigantesca revolución científica y tecnológica. La última década del siglo puede ser resumida en el concepto de globalización, que ha logrado cambiar todos los parámetros de medida durante ese lapso.

\section{Finales de los años 90 y comienzos de la década del 2000}

Los cambios en el contexto social, económico, productivo y educativo han involucrado variaciones normativas, las cuales han impactado en la estructura curricular de las universidades y en su reciprocidad con la incorporación de los nuevos conocimientos del sector agropecuario. Durante esta década, la reducción del financiamiento estatal de la educación obligó a buscar fuentes alternativas de ingresos; esto también contribuyó a un escenario de replanteo y reconfiguración de los roles institucionales tradicionales, al tiempo que generó el acercamiento de las instituciones a la sociedad. En este marco que favoreció a la competitividad y a la globalización, la educación también ha sido atravesada por los fenómenos internacionales. El modelo estadounidense de universidad 86 fue el que mejor se adaptó a nivel internacional y local, debido a que resolvía el problema de la articulación ciencia-tecnología-sociedad y presentó una mejor apertura de la universidad a la sociedad (Barsky y Dávila, 2002).

En este marco, el sistema educativo agropecuario tuvo que enfrentar los cambios que se generaron a partir de los nuevos cultivos, tecnologías y aspectos sociales de la región. Además, las políticas educativas de los últimos años, como los procesos de evaluación y acreditación, fueron una excelente oportunidad para reflexionar acerca del nivel académico que tenían las carreras universitarias y permitieron avanzar en el mejoramiento de la calidad de estas. Para la carrera de Agronomía existen dos mecanismos de acreditación: la nacional y la regional (Mercado Común del Sur [Mercosur], Chile y Bolivia). El proceso de acreditación nacional surge como consecuencia del artículo 43 de la Ley Universitaria $\mathrm{N}^{\circ} 24521$ sancionada y promulgada en 1995. Este artículo dice que los títulos correspondientes a profesiones reguladas por el Estado, cuyo ejercicio pudiera comprometer el interés público y poner en riesgo de modo directo la salud, la seguridad, los derechos, los bienes o la formación de los habitantes, requerirán los siguientes requisitos: 1) una carga horaria mínima; 2) un plan de estudio con contenidos curriculares básicos y criterios sobre intensidad de la formación práctica, y 3) las carreras deberán someterse a periódicos procesos de evaluación y acreditación.

Según el texto elaborado por el IICA en 1998, algunos principios generales a los 
cuales tuvieron que encolumnarse los estudios agronómicos fueron acortar y flexibilizar curricularmente las carreras de grado para lograr un reingreso más rápido de los egresados a las actividades de formación permanente, para que se mantengan actualizados en situaciones de cambio; aumentar la vinculación con el medio y sus demandas; generar la promoción de las actividades y servicios que desarrollan las facultades; desarrollar una visión integradora en el abordaje y solución de los problemas del agro; mejorar la calidad docente a través de la vinculación con problemas de sistemas reales del medio agropecuario, forestal y agroindustrial y dotar a las instituciones de un manejo más empresarial y dinámico con mayor facilidad a la agilidad y variación que los cambios en curso originan en todos los ámbitos.

Varios de estos principios presentaron consecuencias curriculares, como el aumento de una formación básica agronómica, que dejaba la formación específica para después del título de grado; el aumento en la formación para el trabajo, una tendencia a acortar la carrera, a pesar de la oposición de estudiantes y colegios profesionales, y mayor énfasis en los aspectos vinculados con la ética profesional. Asimismo, a los cambios anteriores se sumaron transformaciones a nivel de la gestión y la estructura institucional. En algunas universidades se conformaron nuevos departamentos y se generaron nuevas carreras (Lagomarsino y Amado de Fernández, 2012).
En general, la modalidad más frecuente utilizada para cambiar los planes de estudio durante la década de los 90 se centró en la reestructuración, en aparentes reformulaciones o versiones de un mismo plan de estudio, lo que resultó solamente en una reubicación, un aumento o disminución de materias (Barsky y Davila, 2002). Además, se realizó la estructuración de las asignaturas por años o por ciclos; se modificó el régimen de correlatividades; se suprimieron o agregaron contenidos y finalmente cambiaron la denominación de las materias. Todos estos cambios fueron justificados con la necesidad de actualización. Estas reformulaciones fueron encaradas muchas veces en formas simplistas que lograron distorsionar y ocultar la multiplicidad de variables intervinientes en el proceso de cambio curricular.

La implementación de las reformas curriculares planteadas anteriormente enfrentó dificultades internas de la universidad, hecho que se reflejó en resistencias al ajuste y a los cambios que el sector dominante consideraba necesario, pero a su vez, no se ha resistido a otros (por ejemplo, incentivos para docentes investigadores). La defensa de sectores dentro de las cátedras, el rechazo a la integración de estas o a la realización de cursos entre varias de ellas y la renuencia de docentes y estudiantes a algunas modificaciones curriculares o académicas existieron durante el proceso y aunque no lograron frenar las reformas pudieron cambiar el ritmo 
de avance hacia la extrema lentitud en algunos casos. El cambio institucional en algunas universidades fue fundamental para los cambios curriculares, las reformas académicas y las reformas curriculares-administrativas.

\section{Visión a futuro de la disciplina agropecuaria en relación con los cambios en las últimas décadas}

El contexto actual del sector agropecuario presenta varias tendencias: una marcada globalización económica, tecnológica y productiva, el cuestionamiento de los efectos sociales y ambientales, los nuevos requerimientos alimenticios basados en crecientes exigencias de calidad, diversidad y procesamiento, la multiplicidad de la oferta y el permanente ascenso de la competencia, la crisis de las bases energéticas de la sociedad industrial, entre otras. El proceso actual de globalización debe contemplar la importancia de las diferentes maneras de producir y para esto se deben tener en cuenta los cambios en el conocimiento y en la investigación. Actualmente, son necesarias diferentes capacidades para trabajar y competir en ambientes multiculturales. En este marco, se debe lograr el desarrollo sustentable, con un enfoque sistémico, encaminado a la solución de problemas del sector agrícola, a la seguridad alimentaria y se debe contribuir con investigaciones 88 que posibiliten la disminución de la pobreza en la sociedad rural (Ramírez y Leyva Flores, 2004). Las tendencias actuales hacen necesaria la elaboración de manera participativa de un plan de desarrollo integral de la facultad, que defina su misión, visión y objetivos, revalorizando la docencia, la investigación y la extensión, así como el mejoramiento de la calidad de los procesos técnicos y administrativos internos. Asimismo, es necesaria la construcción de conocimientos diferentes a los que se encuentran actualmente en las carreras de agronomía. Para lograr que estos cambios en los planes de estudio sean consensuados por todos los actores, es necesaria una mayor participación de la comunidad que intervenga durante el proceso y que permita instrumentar y consensuar las transformaciones institucionales, $y$ así responder a las tendencias actuales en políticas educativas.

Los planes de estudio de agronomía evolucionaron del hecho de privilegiar la adquisición de información a una concepción constructivista tendiente al cambio de rol del alumno, de sujeto pasivo a sujeto activo. En relación con las nuevas tendencias en políticas educativas en América latina y su influencia en los planes de estudio de agronomía, varios autores destacan que se deberían lograr currículos significativos y contextualizados para los alumnos y que construyan un puente entre el conocimiento entre los saberes locales y los universales, sin erosionar la identidad de cada región. Estas características hasta el momento han sido difíciles de incluir en los planes de estudio. 


\section{Referencias}

Balsa, J. (2000). Transformaciones en los modos de vida de los productores rurales medios y su impacto en las formas de producción en el agrobonaerense. Actas de las XVII Jornadas de Historia Económica. Universidad Nacional de Tucumán, San Miguel de Tucumán, Argentina.

Barsky, O. (1988). La problemática agraria. Nuevas aproximaciones. Vol. III. Buenos Aires: Centro Editor de América Latina.

Barsky, O. y Dávila, M. (2002). Las transformaciones del sistemainternacional de Educación Superior. Documento de Trabajo N. 93, Universidad de Belgrano. Recuperado de http://www.ub.edu.ar/ investigaciones/dt_nuevos/93_barsky.pdf

Braslavsky, C. y Tiramonti, G. (1990). Conducción educativa y calidad de la enseñanza media. Buenos Aires: Miño y Dávila.

Brunner, J. J. (1990). Educación Superior en América Latina. Cambios y desafios. Chile: Fondo de Cultura Económica.

Carciofi, R. (1987). Educación y aparato productivo en la Argentina, 1976-1982. Un balance de los estudios existentes. En J. C. Tedesco, C. Braslavsky y R. Cardiofi. El proyecto educativo autoritario. 1976-1982 (pp. 175 -223). Buenos Aires: Miño y Dávila Editores.

Craviotti, C. (2000). Las estrategias de los productores familiares ante el cambio tecnológico en la región pampeana argentina. Realidad Económica, (174), 153-161.

Filmus, D. (1999). Estado, sociedad y educación en la Argentina de fin de siglo. Proceso y desafíos, Buenos Aires: Troquel.
García Delgado, D. (1994). Estado y sociedad. Buenos Aires: Norma.

Instituto Interamericano de Cooperación para la Agricultura (IICA) (1998). La reforma curricular en agronomía en la Argentina. Foro de Análisis de la Educación Superior Agropecuaria, Buenos Aires, Argentina

Lagomarsino, E. D. y Amado de Fernández, M. E. (2010). La historia de los procesos de cambio curricular en la Facultad de Agronomía y Zootecnia de la universidad nacional de Tucumán, desde el año 1947 al año 2004. Cuadernos Técnicos. Recuperado el http://www. archivo.unt.edu.ar/attachments/059_ lagomarsinoamado.pdf

Puigross, R. (1973). Universidad peronismo y revolución. Ciencia Nueva, (25), 3-6. Recuperado de http://www. politicascti.net/images/ciencianueva/ CIENCIANUEVA25.pdf

Puiggrós A. (1990). Sujetos, disciplina y currículo en los orígenes del sistema educativo argentino. Buenos Aires: Galerna.

Rama, G. (1987). Estilos educacionales. En D. Saviani (Ed.), Desenvolvimiento y educación en América Latina. Sao Paulo. Cortez Editora.

Ramírez, L. V y Leyva Flores, G. J. F. (20049. Pertinencia social, evaluación y acreditación del agrónomo mexicano. Tiempo de Educar, 5(10), 113-134.

Tedesco, J. C. (1986). Educación y sociedad en Argentina, 1880-1945. Buenos Aires: Ediciones Solar.

Tenti Fanfani, E. (2001). Sociología de la educación. Buenos Aires: Universidad Nacional de Quilmes. 